\title{
ARTICLE
}

\section{Benchmark test of JENDL-4.0 with TOF experiments at Osaka Univ./OKTAVIAN}

Yoshinari Kato $^{\mathrm{a}}$, Kosuke Takakura ${ }^{\mathrm{b} *}$, Masayuki Ohta ${ }^{\mathrm{b}}$, Kentaro Ochiai ${ }^{\mathrm{b}}$, Satoshi Sato ${ }^{\mathrm{b}}$ and Chikara Konno ${ }^{\mathrm{b}}$

${ }^{a}$ Tokai University, 4-1-1 Kitakaname, Hiratsuka-shi, Kanagawa-ken, 259-1292, Japan; ${ }^{b}$ Japan Atomic Energy Agency, 2-4 Shirakata, Tokai-mura, Naka-gun, Ibaraki-ken, 319-1195, Japan

JENDL-4.0, the major revised version of Japanese Evaluated Nuclear Data Library (JENDL), was released in May 2010. In order to validate JENDL-4.0, we analyzed TOF experiments with DT neutrons at OKTAVIAN in Osaka University with JENDL-4.0 and MCNP5.14. For comparison, the older version JENDL-3.3 and other recent nuclear data libraries ENDF/B-VII.0 and JEFF-3.1 were also used. As a result, the followings were found out: (1) Si, As, Se, Mo and W : the calculation results with JENDL-4.0 agreed with the measured ones better than those with JENDL-3.3, (2) $\mathrm{Cr}, \mathrm{Mn}$ and $\mathrm{Nb}$ : the calculation results with JENDL-4.0 were partially better and partially worse than those with JENDL-3.3.

\section{Keywords: JENDL-4.0; benchmark test; MCNP; OKTAVIAN; TOF experiment; DT neutron}

\section{Introduction}

JENDL-4.0 [1], the major revised version of Japanese Evaluated Nuclear Data Library (JENDL), was released in May, 2010. It is important to validate JENDL-4.0 through analyses of integral benchmark experiments. So far we benchmarked it through the benchmark experiments carried out at the Fusion Neutronics Source (FNS) facility in Japan Atomic Energy Agency (JAEA)
$[2,3]$ on beryllium, carbon, silicon, vanadium, iron, copper, tungsten and lead.

From 1984 to 1988 , sphere pile integral benchmark experiments were carried out with DT neutrons of OKTAVIAN in Osaka University [4] on $\mathrm{LiF}, \mathrm{CF}_{2}, \mathrm{Al}, \mathrm{Si}$, $\mathrm{Ti}, \mathrm{Cr}, \mathrm{Mn}, \mathrm{Co}, \mathrm{Cu}, \mathrm{As}, \mathrm{Se}, \mathrm{Zr}, \mathrm{Nb}, \mathrm{Mo}$ and W. Thus we analyze these experiments with JENDL-4.0 as one of benchmark tests of JENDL-4.0.

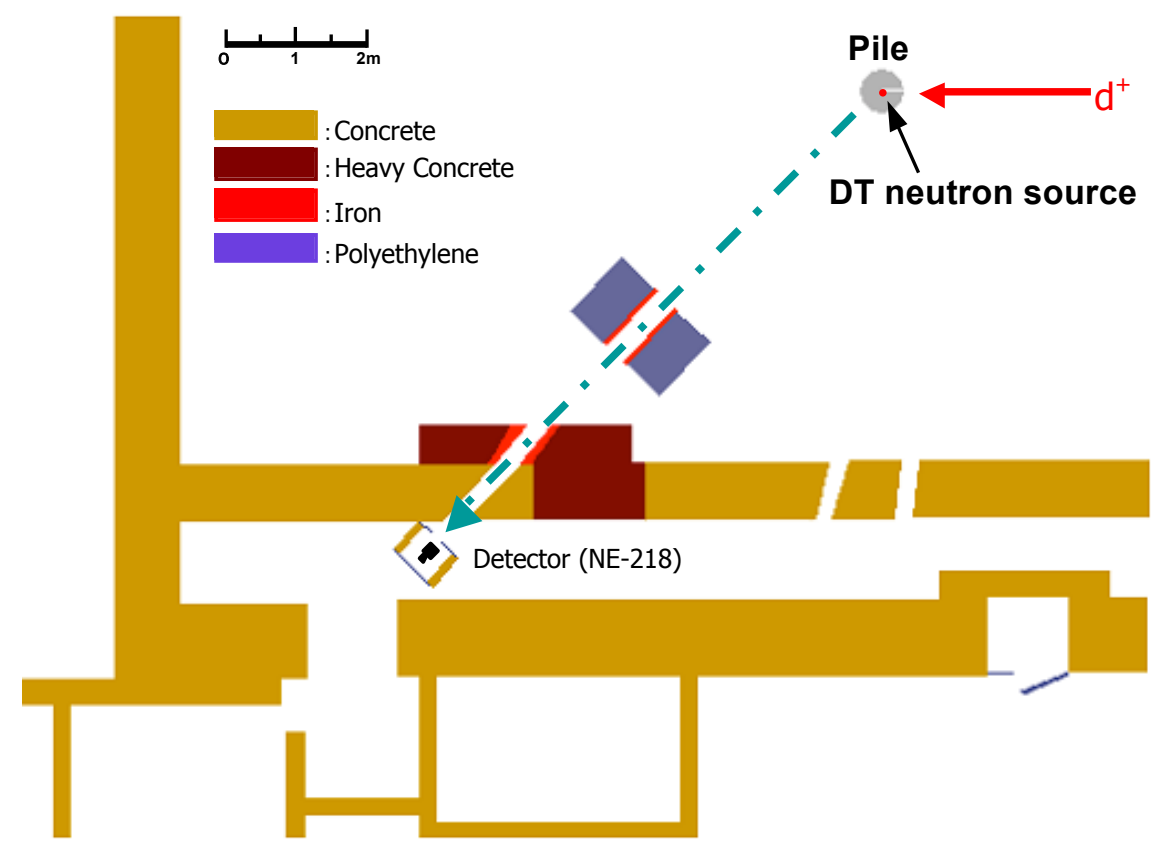

Figure 1. Experimental arrangement.

*Corresponding author. Email: takakura.kosuke@jaea.go.jp 


\section{Overview of TOF experiments at OKTAVIAN}

Leakage neutron spectra from spherical piles were measured with the time-of flight (TOF) technique at the DT neutron source facility OKTAVIAN in Osaka University. The piles were made by filling spherical vessels with sample powder or flakes of $\mathrm{LiF}, \mathrm{CF}_{2}, \mathrm{Al}, \mathrm{Si}$, Ti, Cr, Mn, Co, Cu, As, Se, Zr, Nb, Mo and W. The material densities and outer diameters of the vessels are summarized in Table 1. DT neutrons were produced by bombarding a $370 \mathrm{GBq}$ tritium target placed at the center of the pile with $250 \mathrm{keV}$ deuteron beam. A cylindrical liquid organic scintillator NE-218 was used as a neutron detector, which was located at about $11 \mathrm{~m}$ from the tritium target and $55 \mathrm{deg}$. with respect to the deuteron beam axis, surrounded by concrete and heavy concrete. A pre-collimator made of polyethylene-iron multi-layers was set between the pile and the detector in order to reduce the background neutrons. The experimental arrangement is shown in Figure 1.

Table 1. Material density and outer diameter of vessel.

\begin{tabular}{ccc}
\hline Material & $\begin{array}{c}\text { Apparent density } \\
\left(\mathrm{g} / \mathrm{cm}^{3}\right)\end{array}$ & $\begin{array}{c}\text { Outer diameter of vessel } \\
(\mathrm{cm})\end{array}$ \\
\hline $\mathrm{LiF}$ & 1.79 & 61 \\
$\mathrm{CF}_{2}$ & 1.30 & 40 \\
$\mathrm{Al}$ & 1.22 & 40 \\
$\mathrm{Si}$ & 1.29 & 60 \\
$\mathrm{Ti}$ & 1.54 & 40 \\
$\mathrm{Cr}$ & 3.72 & 40 \\
$\mathrm{Mn}$ & 4.37 & 61 \\
$\mathrm{Co}$ & 1.94 & 40 \\
$\mathrm{Cu}$ & 6.23 & 61 \\
$\mathrm{As}$ & 3.09 & 40 \\
$\mathrm{Se}$ & 2.29 & 40 \\
$\mathrm{Zr}$ & 2.84 & 61 \\
$\mathrm{Nb}$ & 4.39 & 28 \\
$\mathrm{Mo}$ & 2.15 & 61 \\
$\mathrm{~W}$ & 4.43 & 40 \\
\hline
\end{tabular}

\section{Analysis}

The Monte Carlo code MCNP-5.14 [5] and the official ACE file FSXLIB-J40 of JENDL-4.0 were used for the analysis. Calculations with the ACE files of the following nuclear data libraries were also carried out for comparison.

-JENDL-3.3 (ACE file : FSXLIB-J33) [6]

-ENDF/B-VII.0 (ACE file : endf70 in MCNP Data) [7]

-JEFF-3.1 (ACE file : MCJEFF3.1) [8]

Experiments for lithium fluoride and aluminum piles were not analyzed in this work because the data of these nuclei were not revised in JENDL-4.0.

\section{Results and discussion}

\section{1. $\mathrm{CF}_{2}$ sphere}

The measured and calculated neutron spectra are shown in Figure 2(a) with ratios of calculation to experiment $(\mathrm{C} / \mathrm{E})$ for each specific energy region of 0.1-0.5 MeV, 0.5-1 MeV, 1-5 MeV, 5-10 MeV and > 10 $\mathrm{MeV}$. There is no difference between the calculation results with JENDL-3.3 and JENDL-4.0, though the capture cross-section data of carbon are modified in JENDL-4.0. All the calculation results underestimated the measured one by $\sim 40 \%$. The measured neutron spectrum may have some problems.

\subsection{Si sphere}

The $\mathrm{Si}$ isotope data in JENDL-4.0 are newly evaluated by using the TNG code [9]. The resolved resonance parameters are taken from ENDF/B-VII.0. Thus there are many modifications in the $\mathrm{Si}$ isotope data of JENDL-4.0. Figure 2(b) shows the measured and calculated neutron spectra with $\mathrm{C} / \mathrm{Es}$ for each specific energy region. The underestimation around $8 \mathrm{MeV}$ in the calculation result with JENDL-3.3 is improved in that with JENDL-4.0, though the difference between the calculation results with JENDL-3.3 and JENDL-4.0 was

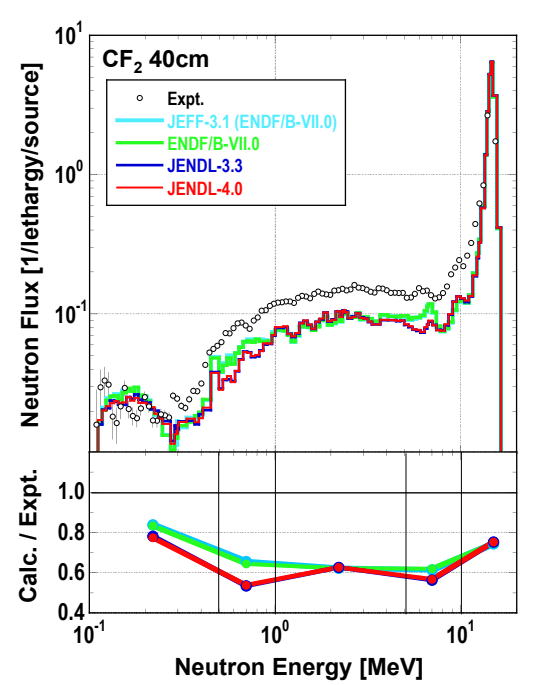

(a) $\mathrm{CF}_{2}$ sphere

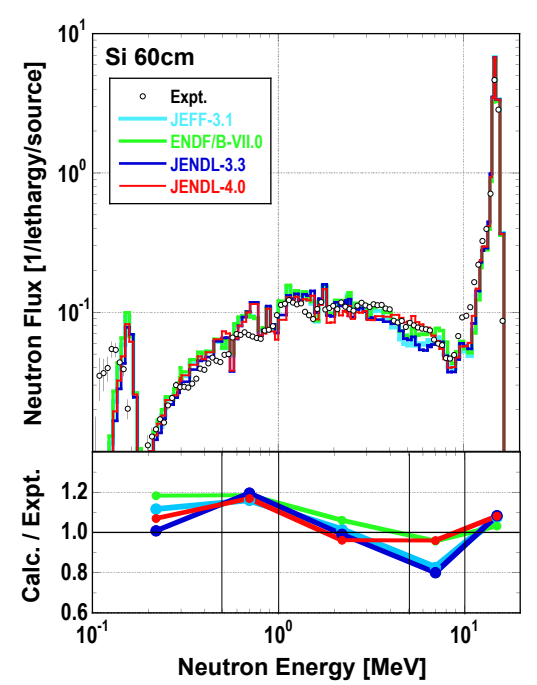

(b) Si sphere

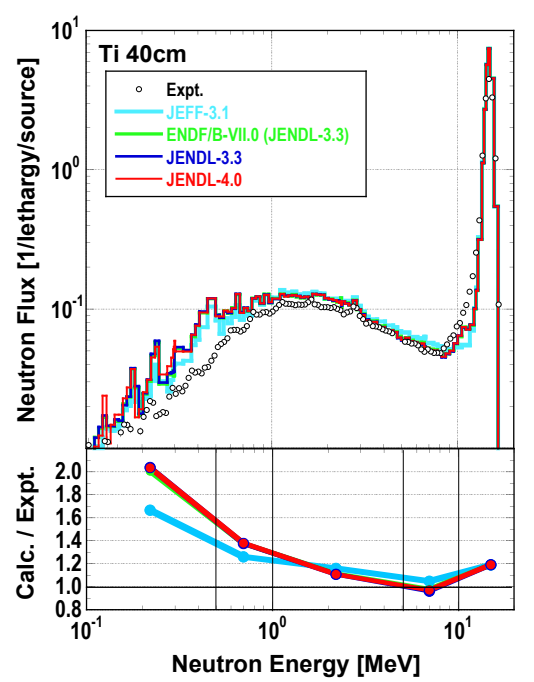

(c) Ti sphere

Figure 2. Measured and calculated leakage neutron spectra from sphere of (a) $\mathrm{CF}_{2}$, (b) $\mathrm{Si}$ and (c) Ti. 
small in the $\mathrm{SiC}$ in-situ experiment at JAEA/FNS [3]. The calculation result with JENDL-4.0 is better than those with ENDF/B-VII.0 and JEFF-3.1.

\subsection{Ti sphere}

The resolved resonance parameters of the titanium isotopes are modified in JENDL-4.0. Figure 2(c) shows the measured and calculated neutron spectra with $\mathrm{C} / \mathrm{Es}$ for each specific energy region. The calculation result with JENDL-4.0 is almost the same as that with JENDL-3.3. The overestimation below $1 \mathrm{MeV}$ is not improved.

\subsection{Cr sphere}

The data above the resolved resonance region of the chromium isotopes in JENDL-4.0 are re-evaluated with the CCONE code [10]. Figure 3(a) shows the measured and calculated neutron spectra with $\mathrm{C} / \mathrm{Es}$ for each specific energy region. The calculation result with JENDL-4.0 tends to overestimate the measured one more than that with JENDL-3.3, particularly around 8 $\mathrm{MeV}$. The chromium data in JENDL-3.3 are the best.

\subsection{Mn sphere}

The resolved resonance parameters and the elastic scattering cross section data of ${ }^{55} \mathrm{Mn}$ are modified in JENDL-4.0. Moreover the unresolved resonance data are newly added in JENDL-4.0. Figure 3(b) shows the measured and calculated neutron spectra with $\mathrm{C} / \mathrm{Es}$ for each specific energy region. The calculation result with JENDL-4.0 overestimates the measured one below 1 $\mathrm{MeV}$ more than that with JENDL-3.3. This is considered to be due to the unresolved resonance data newly added in JENDL-4.0 [11].

\subsection{Co sphere}

Figure 3(c) shows the measured and calculated neutron spectra with $\mathrm{C} / \mathrm{Es}$ for each specific energy region. The calculation result with JENDL-4.0 is almost the same as that with JENDL-3.3. The underestimation

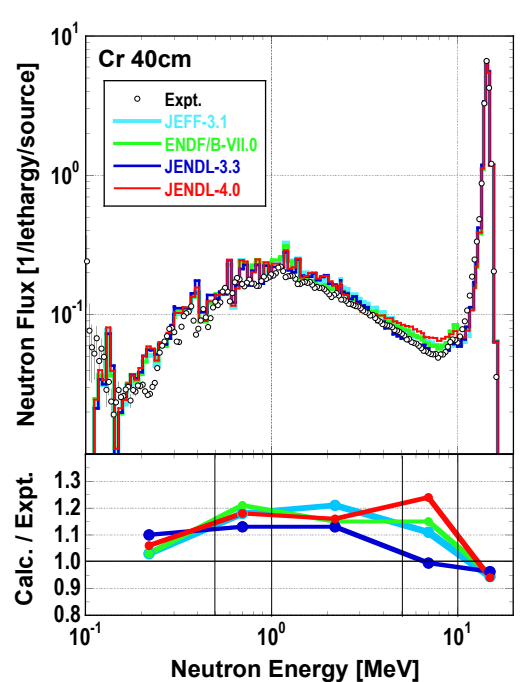

(a) Cr sphere

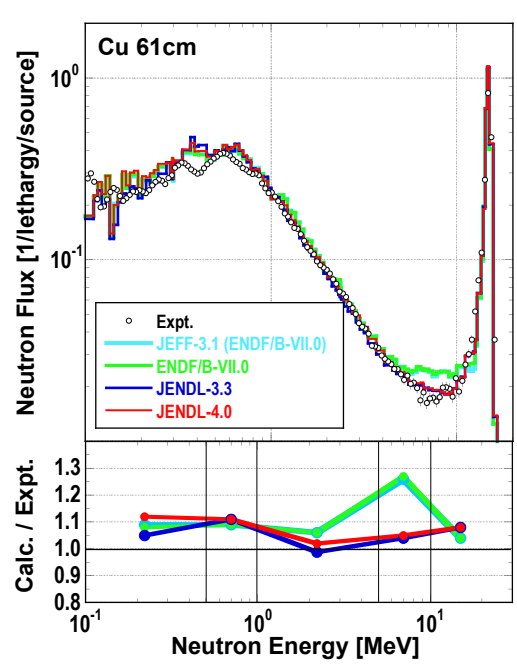

(d) $\mathrm{Cu}$ sphere

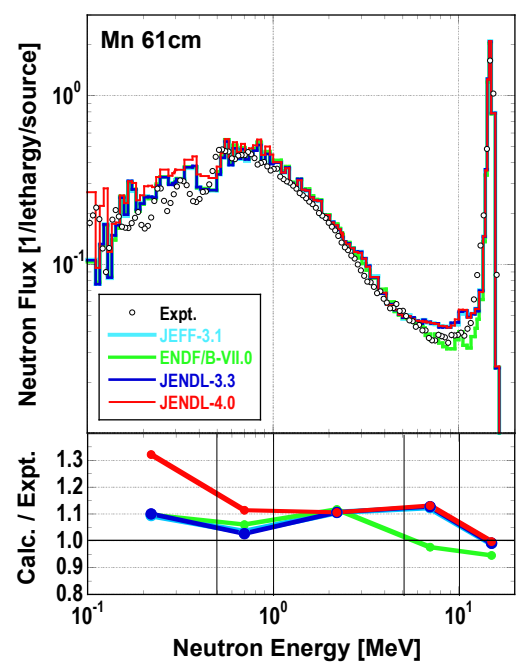

(b) Mn sphere

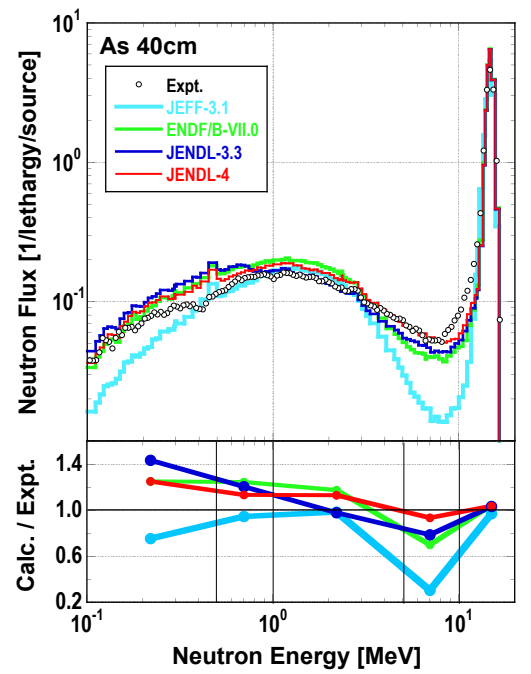

(e) As sphere

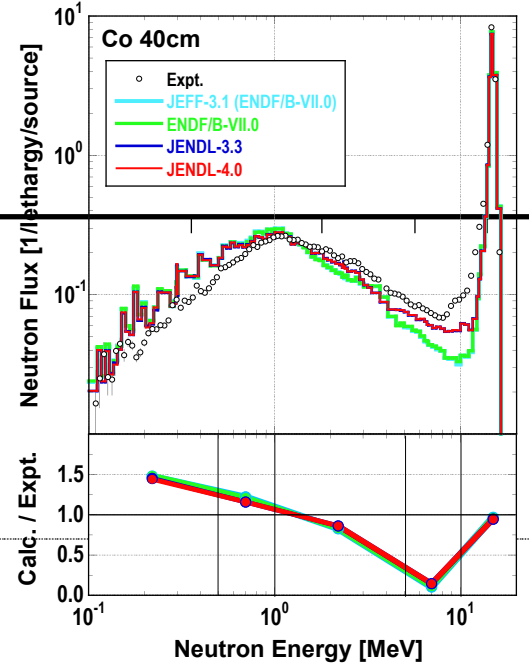

(c) Co sphere

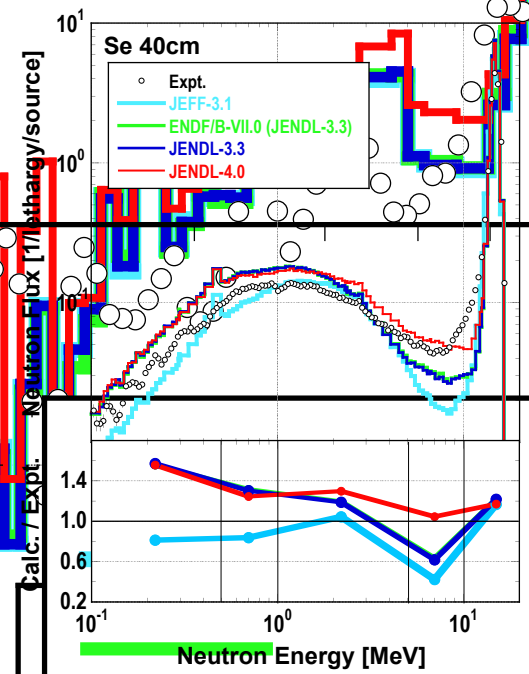

(f) Se sphere

Figure 3. Measured and calculated leakage neutron spectra from sphere of (a) Cr, (b) $\mathrm{Mn}$, (c) Co, (d) Cu, (e) As and (f) Se. 
from a few $\mathrm{MeV}$ to $10 \mathrm{MeV}$ and overestimation below 1 $\mathrm{MeV}$ are not improved at all. Those with ENDF/B-VII.0 and JEFF-3.1 are slightly worse than those with JENDL-3.3 and JENDL-4.0.

\subsection{Cu sphere}

The cross section and angular distribution data of the elastic scattering in ${ }^{63} \mathrm{Cu}$ and ${ }^{65} \mathrm{Cu}$ are modified in JENDL-4.0. The measured and calculated neutron spectra are shown in Figure 3(d) with $\mathrm{C} / \mathrm{Es}$ for each specific energy region. The calculation result with JENDL-4.0 overestimates the measured one slightly compared to that with JENDL-3.3, though it is better than those with ENDF/B-VII.0 and JEFF-3.1.

\subsection{As sphere}

All the data of ${ }^{75} \mathrm{As}$ in JENDL-4.0 are re-evaluated with the POD code [12]. Figure 3(e) shows the measured and calculated neutron spectra with $\mathrm{C} / \mathrm{Es}$ for each specific energy region. The underestimation from 3 $\mathrm{MeV}$ to $10 \mathrm{MeV}$ and overestimation below $1 \mathrm{MeV}$ in the calculation result with JENDL-3.3 are improved in that with JENDL-4.0, while the calculation result with JENDL-4.0 overestimates the measured neutron flux from $1 \mathrm{MeV}$ to $3 \mathrm{MeV}$. Generally speaking, the calculation result with JENDL-4.0 is the best.

\subsection{Se sphere}

Most data of the selenium isotopes in JENDL-4.0 are re-evaluated with the POD code. Figure 3(f) shows the measured and calculated neutron spectra with $\mathrm{C} / \mathrm{Es}$ for each specific energy region. The underestimation of the neutron flux from $3 \mathrm{MeV}$ to $10 \mathrm{MeV}$ is improved in the calculation result with JENDL-4.0. The overestimation below $1 \mathrm{MeV}$ is not improved even in the calculation result with JENDL-4.0.

\subsection{Zr sphere}

The resonance parameters and the capture cross-section data of the zirconium isotopes are modified in JENDL-4.0. Figure 4(a) shows the measured and calculated neutron spectra with $\mathrm{C} / \mathrm{Es}$ for each specific energy region. The calculation result with JENDL-4.0 is almost the same as that with JENDL-3.3.

\subsection{Nb sphere}

The resonance parameters and the energy-angle distribution data of ${ }^{93} \mathrm{Nb}$ are mainly modified in JENDL-4.0. Figure 4(b) shows the measured and calculated neutron spectra with $\mathrm{C} / \mathrm{Es}$ for each specific energy region. The calculation result with JENDL-4.0 overestimates the measured neutron flux from $0.7 \mathrm{MeV}$ to $10 \mathrm{MeV}$, while that with JENDL-3.3 agrees with the measured neutron flux well. The overestimation below 1 $\mathrm{MeV}$ is slightly improved in the calculation result with JENDL-4.0. The calculation result with ENDF/B-VII.0 (JEFF-3.1) overestimates the measured neutron flux from $0.6 \mathrm{MeV}$ to $10 \mathrm{MeV}$ more, though it agrees with the measured neutron flux below $0.6 \mathrm{MeV}$.

\subsection{Mo sphere}

Most data of the molybdenum isotopes in JENDL-4.0 are re-evaluated with the POD code. Figure 4(c) shows the measured and calculated neutron spectra with $\mathrm{C} / \mathrm{Es}$ for each specific energy region. The calculation result with JENDL-4.0 agrees with the measured neutron flux from $0.6 \mathrm{MeV}$ to $5 \mathrm{MeV}$ better than those with JENDL-3.3, ENDF/B-VII.0 and JEFF-3.1, while it slightly underestimates the measured neutron flux below $0.5 \mathrm{MeV}$.

\subsection{W sphere}

Most data of the tungsten isotopes in JENDL-4.0 are

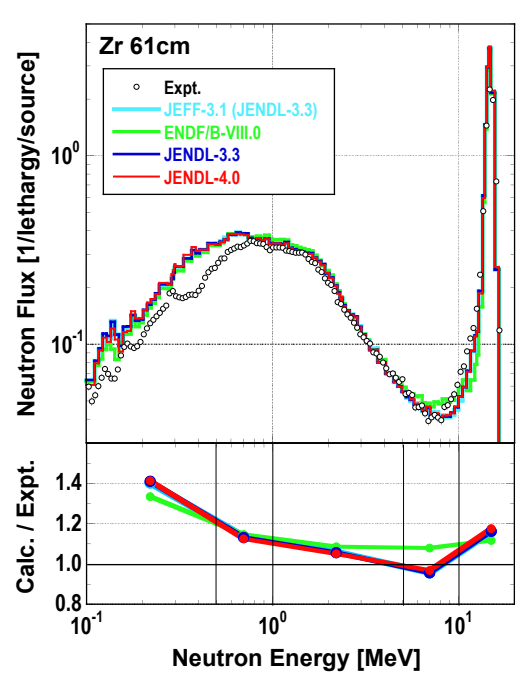

(a) Zr sphere

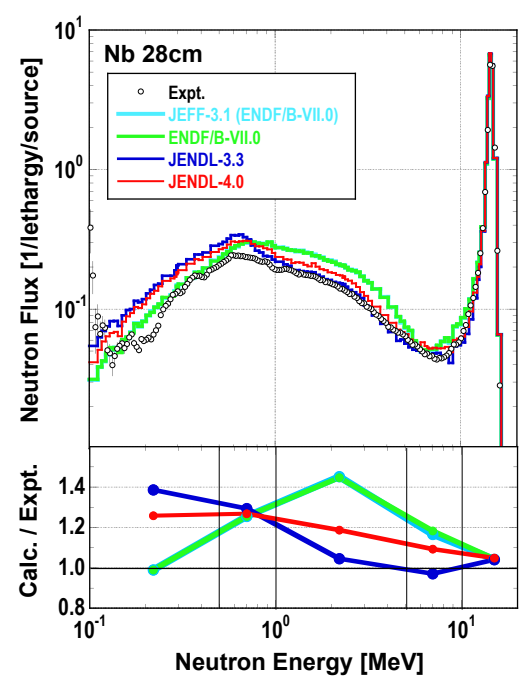

(b) $\mathrm{Nb}$ sphere

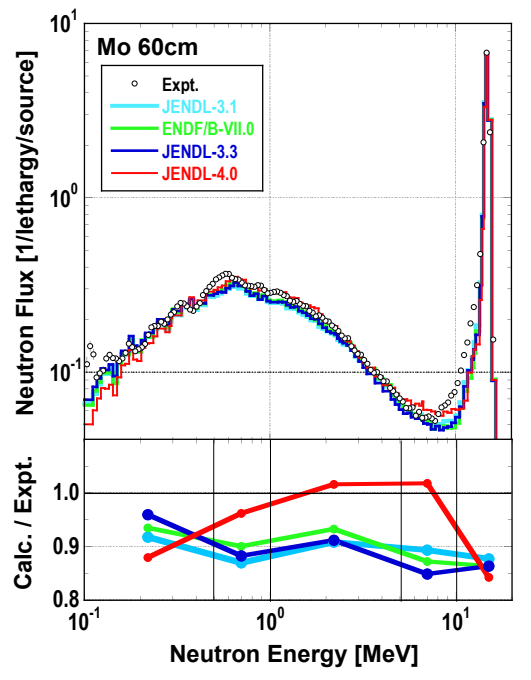

(c) Mo sphere

Figure 4. Measured and calculated leakage neutron spectra from sphere of (a) $\mathrm{Zr}$, (b) $\mathrm{Nb}$ and (c) Mo. 
re-evaluated with the CCONE code. Figure 5 shows the measured and calculated neutron spectra with $\mathrm{C} / \mathrm{Es}$ for each specific energy region. The calculation result with JENDL-4.0 agrees with the measured one better than that with JENDL-3.3 and it is almost the same as that with ENDF/B-VII.0.

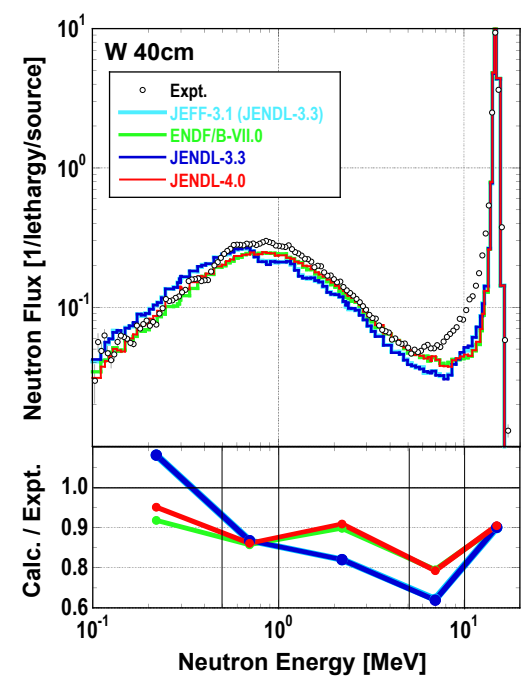

Figure 5. Measured and calculated leakage neutron spectra from $\mathrm{W}$ sphere.

\section{Conclusion}

We benchmarked JENDL-4.0 with the TOF experiments at Osaka University / OKTAVIAN. The followings are found out:

1) $\mathrm{Si}, \mathrm{As}, \mathrm{Se}, \mathrm{Mo}$ and $\mathrm{W}$ spheres : the calculation results with JENDL-4.0 agree with the measured ones better than those with JENDL-3.3.

2) $\mathrm{CF}_{2}, \mathrm{Ti}, \mathrm{Co}, \mathrm{Cu}$ and $\mathrm{Zr}$ spheres : the calculation results with JENDL-4.0 are comparable to those with JENDL-3.3.

3) $\mathrm{Cr}, \mathrm{Mn}$ and $\mathrm{Nb}$ spheres : the calculation results with JENDL-4.0 are partially better and partially worse than those with JENDL-3.3.

It is generally concluded that JENDL-4.0 is improved compared to JENDL-3.3. Next we will investigate which reactions in JENDL-4.0 contribute to the improvement or deterioration in detail.

\section{References}

[1] K. Shibata, O. Iwamoto, T. Nakagawa, N. Iwamoto, A. Ichihara, S. Kunieda, S. Chiba, K. Furutaka, N. Otuka, T. Ohsawa, T. Murata, H. Matsunobu, A.
Zukeran, S. Kamada and J. Katakura, JENDL-4.0: A new library for nuclear science and engineering, J. Nucl. Sci. Technol. 48 (2010), pp. 1-30.

[2] C. Konno, K. Takakura, M. Wada, K. Kondo, S. Ohnishi, K. Ochiai and S. Sato, Benchmark test of JENDL-4.0 based on integral experiments at JAEA/FNS, Prog. Nucl. Sci. Tech. 2 (2011), pp. 346-357.

[3] C. Konno, M. Wada, K. Kondo, S. Ohnishi, K. Takakura, K. Ochiai and S. Sato, Detailed benchmark test of JENDL-4.0 iron data for fusion applications, Fusion Eng. Des. 86 (2011), pp. 2682-2685.

[4] F. Maekawa, J. Yamamoto, C. Ichihara, K. Ueki and Y. Ikeda, Collection of experimental data for fusion neutronics benchmark, JAERI-M 94-014, Japan Atomic Energy Research Institute, (1994).

[5] X-5 Monte Carlo Team, MCNP - A General Monte Carlo N-Particle Transport Code, Version 5, LA-UR-03-1987 revised, Los Alamos National Laboratory, (2008).

[6] K. Kosako, N. Yamano, T. Fukahori, K. Shibata and A. Hasegawa, The Libraries FSXLIB and MATXSLIB based on JENDL-3.3, JAERI-Data/Code 2003-011, Japan Atomic Energy Research Institute, (2003).

[7] $M C N P 5 / M C N P X-E X E$, RSICC CODE PACKAGE CCC-740, Oak Ridge National Laboratory, (2009).

[8] O. Cabellos, Processing of the JEFF-3.1 Cross Section Library into a Continuous Energy Monte Carlo Radiation Transport and Criticality Data Library, NEA/NSC/DOC 18, OECD NEA Data Bank, (2006).

[9] K. Shibata and C. Fu, Recent Improvements of the TNG Statistical Model Code, ORNL/TM-10093, Oak Ridge National Laboratory, (1986).

[10]O. Iwamoto, Development of a comprehensive code for nuclear data evaluation, CCONE, and validation using neutron-induced cross sections for uranium isotopes, J. Nucl. Sci. Technol. 44 (2007), pp. 687-697.

[11]C. Konno, Y. Kato, K. Takakura, M. Ohta, K. Ochiai and S. Sato, Self-shielding effect of unresolved resonance data in JENDL-4.0, submitted to proceeding of this conference.

[12]A. Ichihara, O. Iwamoto, S. Chiba, S. Kunieda and K. Shibata, Program POD; A Computer Code to Calculate Cross Sections for Neutron-induced Nuclear Reactions, JAEA-Data/Code 2007-012, Japan Atomic Energy Agency, (2007). 